\title{
Socio-demographic Factors Associated with Home Delivery Assisted by Untrained Traditional Birth Attendant in Rural Bangladesh
}

\author{
Md. Atiqul Hoque Chowdhury 1,*, Md. Mehedi Hasan², Sayem Ahmed², Charls Darwin ${ }^{3}$, Mohammad Sazzad Hasan", \\ Md. Rabiul Haque ${ }^{5}$
}

\author{
${ }^{1}$ Department of Public Health, University of South Asia, Dhaka, Bangladesh \\ ${ }^{2}$ International Centre for Diarrhoeal Disease Research, Bangladesh (icddr,b), Dhaka, Bangladesh \\ ${ }^{3}$ Faculty of Business Studies, Bangladesh University of Professionals, Dhaka, Bangladesh \\ ${ }^{4}$ Department of management studies, Jagannath University, Dhaka, Bangladesh \\ ${ }^{5}$ Department of Population Sciences, University of Dhaka, Dhaka, Bangladesh \\ *Corresponding author: cshamol@yahoo.com
}

Received November 08, 2013; Revised November 28, 2013; Accepted December 08, 2013

\begin{abstract}
Delivery assisted by skilled birth attendant is recommended as a means to reduce maternal mortality. However untrained traditional birth attendant (UTBA) assisted home is very high in developing countries. The objective of this study is to assess socio-demographic determinants of UTBA assisted home delivery in rural Bangladesh. Data used in this study was obtained from women questionnaire of the 2011 Bangladesh Demographic and Health Survey (BDHS). Mothers residing in rural Bangladesh, who gave valid information on both delivery place and assistance during delivery, were included in this study $(n=4983)$. Binary logistic regression model was used to explore factors influencing home delivery assisted by untrained traditional birth attendant. Most of the mothers (79\%) reported to have delivered at home and of these home deliveries, 76\% were assisted by untrained traditional birth attendant. In multivariate regression model, it is found that the odds of UTBA assisted home delivery decreased with mother's education, husband's education, higher wealth index, number of Antenatal Care (ANC) visits and media exposure. To reduce maternal mortality in rural areas of Bangladesh, program managers and policy makers should consider these factors in designing programs and implementing interventions.
\end{abstract}

Keywords: untrained traditional birth attendants, home delivery, Bangladesh

Cite This Article: Md. Atiqul Hoque Chowdhury, Md. Mehedi Hasan, Sayem Ahmed, Charls Darwin, Mohammad Sazzad Hasan, and Md. Rabiul Haque, "Socio-demographic Factors Associated with Home Delivery Assisted by Untrained Traditional Birth Attendant in Rural Bangladesh." American Journal of Public Health Research 1, no. 8 (2013): 226-230. doi: 10.12691/ajphr-1-8-6.

\section{Introduction}

Maternal mortality is a global health concern. Every year, an estimated 287000 women die worldwide from pregnancy related causes, and $99 \%$ of these deaths occur in developing countries [1]. A primary cause of high maternal mortality in developing countries is the absence of skilled care attendants at the time of delivery [2]. Ensuring skilled birth attendants during delivery and in the early postpartum period can lead to marked reductions in maternal mortality even up to $75 \%$ or more $[3,4,5,6]$. In developing countries, home birth is a common phenomenon and a significant proportion of women still deliver at home being assisted by untrained traditional birth attendant (UTBA) [7,8].

Place of delivery with its determinants have long been on the research agenda [9]. In diverse contexts, maternal age, parity, education, family size, household wealth, socio-economic status, place of residence, availability and distance of health facilities etc determine the place of delivery [10,11,12]. UTBA assisted home delivery is associated with young maternal age, low educational attainment, rural residence, low socio-economic status, high birth order, absence of Antenatal care (ANC) services, distance to health facilities, delivery complication etc $[11,12,13]$.

In Bangladesh, maternal mortality rate has decreased over recent years but still remains to be a problem of public health agenda. In rural Bangladesh, most of the deliveries occur at home assisted by untrained traditional birth attendant [14]. Recent evidence on determinants of UTBA assisted home delivery in rural Bangladesh using a nationally representative data is lacking, yet understanding the influences. In rural Bangladesh, it is said that UTBA assisted home delivery is one of the cause of maternal mortality. Very few studies in Bangladesh have focused on the determinants of UTBA assisted home delivery, particularly in rural areas. Thus in rural Bangladesh it is very important to characterize women who are assisted by UTBA during home delivery, for designing specific 
interventions to such groups. The objective of this study is to assess the socio-demographic determinants of UTBA assisted home delivery in rural Bangladesh.

\section{Methodology}

\subsection{Study Population}

The study was conducted using data from Bangladesh Demographic and Health Survey (BDHS) 2011. As part of global Demographic and Health Surveys (DHS) program, the 2011 BDHS is nationally representative household based survey conducted to obtain information on health and demography, and focusing on maternal and child health. Using the 2011 Bangladesh Population and Housing Census, a two stage stratified sampling technique was used to sample 18000 household from 600 clusters throughout Bangladesh, 207 in urban areas and 393 in the rural areas. A total of 17,842 ever married women aged 13-49 years were interviewed. Information obtained in this survey was self-reported. In this study, analysis was restricted to the women who reside in rural areas of Bangladesh and also gave valid information on both delivery place and assistance taken during delivery of their most recent birth $(n=4983)$.

\subsection{Outcome and Explanatory Variables}

"UTBA assisted home delivery" is the outcome variable in this study. If any delivery took place at home with the presence of untrained traditional birth attendant, it was considered as "UTBA assisted home delivery". Based on the review of previous studies, a total of seven explanatory variables were selected for analysis from the women questionnaire, $[8,11,12,15]$. These sociodemographic variables are maternal age, mother's education, husband's education, sex of the household head, wealth index, number of ANC visits and media exposure.

Among the explanatory variables, maternal age was recoded into $<20,20-34,34-49$ years, education into no education, primary education, secondary or higher education, and number of ANC visits into $0,1-3, \geq 4$. The wealth index as a proxy measure of socio-economic status was calculated using data on consumer goods, type of toilet facilities and drinking water source, dwelling characteristics, land possessed etc. Details of the construction of this index are discussed in detail by Measure DHS [16]. Media exposure is a composite variable. A woman is considered as exposed to media if she either watches television or listens to radio or reads newspaper.

\subsection{Statistical Analysis}

Frequencies and proportions were calculated to describe the sample, according to the outcome of interest and other socio-demographic characteristics. In multivariate analysis, binary logistic model was used to explore the factors influencing home delivery assisted by untrained traditional birth attendant. Here, UTBA assisted home delivery was coded as " 1 " and other type of care as " 0 ". The results of multivariate logistic regression model were reported as odds ratio (OR) with their 95\% confidence intervals (CI). All the analyses were done in STATA using 'svy' commands to allow the adjustments of cluster effect and sampling weight.

\subsection{Ethical Consideration}

Data used in this study was de-identified data obtained from MEASURE DHS. Ethical approval was not necessary, as the study was conducted on anonymous public use data having no identifiable information on the survey respondents.

\section{Results}

Mothers residing in rural Bangladesh, who gave valid information on both delivery place and assistance during delivery, were included in our study ( $n=4983)$. Most of the women (79\%) delivered their most recent birth at home and of these home deliveries $76 \%$ were assisted by untrained traditional birth attendant (table not shown). Table 1 represents the background characteristics of the respondents. Among the mothers, more than half of the women $(68.7 \%)$ were of age 25-34 years and about 1 in 5 women (21\%) had no education. Fifty seven percent women were exposed to mass media and about 1 in 5 women (19\%) received 4 or more antenatal care services during their last pregnancy.

Table 1. Distribution of socio-demographic characteristics of the respondents

\begin{tabular}{|c|c|c|}
\hline Characteristics & $\mathrm{N}$ & $\%$ \\
\hline \multicolumn{3}{|l|}{ Maternal age } \\
\hline$<20$ & 1289 & 25.87 \\
\hline $20-34$ & 3397 & 68.17 \\
\hline $35-49$ & 297 & 5.69 \\
\hline \multicolumn{3}{|l|}{ Mother's education } \\
\hline No education & 1041 & 20.89 \\
\hline Primary & 1567 & 31.45 \\
\hline Secondary or higher & 2375 & 47.66 \\
\hline \multicolumn{3}{|l|}{ Husband's education } \\
\hline No education & 1532 & 30.78 \\
\hline Primary & 1507 & 30.27 \\
\hline Secondary or higher & 1939 & 38.95 \\
\hline \multicolumn{3}{|l|}{ Sex of the household head } \\
\hline Female & 399 & 8.01 \\
\hline Male & 4584 & 91.99 \\
\hline \multicolumn{3}{|l|}{ Wealth index } \\
\hline Poorest & 1315 & 26.39 \\
\hline Poorer & 1221 & 24.50 \\
\hline Middle & 1116 & 22.40 \\
\hline Richer & 869 & 17.44 \\
\hline Richest & 462 & 9.27 \\
\hline \multicolumn{3}{|l|}{ No. of ANC visits } \\
\hline 0 & 2000 & 40.16 \\
\hline $1-3$ & 2051 & 41.18 \\
\hline $4+$ & 929 & 18.65 \\
\hline \multicolumn{3}{|l|}{ Media exposure } \\
\hline No & 2157 & 43.29 \\
\hline Yes & 2826 & 56.71 \\
\hline
\end{tabular}


Table 2 presents factors associated with UTBA assisted home deliveries that were considered in the multivariate logistic regression analysis. Mother's education, husband's education, wealth index, number of ANC visits and media exposure were significantly associated with untrained TBA assisted home delivery. Maternal age and Sex of the household head were not significantly associated with UTBA assistance during home delivery.

Women having secondary or higher education were 0.723 times less likely to be delivered at home by UTBA compared to those who had no education (OR=0.723, 95\% CI:0.577-0.907). Women whose husband had secondary or higher education were 0.691 times less likely to be assisted by UTBA during home delivery than whose husbands had no education (OR=0.691, 95\% CI: 0.571 0.837). Women in highest wealth quintile were 0.557 times less likely to be delivered at home by UTBA compared to the women in the lowest wealth quintile (OR=0.557, 95\% CI: 0.424-0.731). Women who received four or more ANC visits were 0.267 times less likely to be delivered at home than the women who didn't received any ANC (OR=0.267, 95\% CI:0.219-0.326). Women who were exposed to mass media were 0.805 times less likely to deliver at home by UTBA compared to those who were not exposed to media (OR=0.805, 95\% CI: 0.701-0.925).

Table 2. Odds Ratio (OR) and 95\% confidence interval (CI) of the association of socio-demographic variables and untrained TBA assisted home delivery in rural Bangladesh

\begin{tabular}{|c|c|c|c|c|}
\hline \multirow{2}{*}{ Characteristics } & \multirow{2}{*}{ Odds Ratio (OR) } & \multicolumn{2}{|c|}{ 95\% Confidence Interval (CI) } & \multirow{2}{*}{ Sig. } \\
\hline & & Lower & Upper & \\
\hline \multicolumn{5}{|l|}{ Maternal age } \\
\hline$<20$ & 1 & & & \\
\hline $20-34$ & 1.001 & 0.864 & 1.159 & 0.998 \\
\hline $35-49$ & 0.889 & 0.652 & 1.214 & 0.460 \\
\hline \multicolumn{5}{|l|}{ Mother's education } \\
\hline No education & 1 & & & \\
\hline Primary & 1.034 & 0.845 & 1.265 & 0.745 \\
\hline Secondary or higher & 0.723 & 0.577 & 0.907 & 0.005 \\
\hline \multicolumn{5}{|l|}{ Husband's education } \\
\hline No education & 1 & & & \\
\hline Primary & 0.922 & 0.765 & 1.112 & 0.395 \\
\hline Secondary or higher & 0.691 & 0.571 & 0.837 & 0.000 \\
\hline \multicolumn{5}{|c|}{ Sex of the household head } \\
\hline Female & 1 & & & \\
\hline Male & 0.927 & 0.733 & 1.171 & 0.522 \\
\hline \multicolumn{5}{|l|}{ Wealth index } \\
\hline Poorest & 1 & & & \\
\hline Poorer & 1.156 & 0.958 & 1.395 & 0.130 \\
\hline Middle & 0.963 & 0.780 & 1.189 & 0.724 \\
\hline Richer & 0.752 & 0.605 & 0.935 & 0.010 \\
\hline Richest & 0.557 & 0.424 & 0.731 & 0.000 \\
\hline \multicolumn{5}{|l|}{ No. of ANC visits } \\
\hline 0 & 1 & & & \\
\hline $1-3$ & 0.508 & 0.439 & 0.587 & 0.000 \\
\hline $4+$ & 0.267 & 0.219 & 0.326 & 0.000 \\
\hline \multicolumn{5}{|l|}{ Media exposure } \\
\hline No & 1 & & & \\
\hline Yes & 0.805 & 0.701 & 0.925 & 0.002 \\
\hline
\end{tabular}

\section{Discussion}

This study of rural Bangladeshi women at their most recent delivery, reported that most of the women delivered at home and most of these home deliveries were assisted by UTBA. This proportion of UTBA assisted home delivery is higher than both urban areas and national level in Bangladesh. Since health service facility and social environment are different in rural-urban setting, strong rural-urban differences in the use of delivery care are expected [17]. High proportion of home delivery in rural areas may be due to low education, less ability to pay, high parity, lack of delivery care information, low availability and accessibility of services, poor infrastructure and communication. Socio-demographic factors such as mother's education, husband's education, wealth index, number of ANC visits, and media exposure, were found statistically significant with UTBA assisted home delivery. Maternal age and Sex of the household head were not significantly associated with UTBA assisted home delivery.

More than half of the women have no secondary or higher education. Poverty and early marriage may be the major causes that stop rural women from completing secondary or higher educations. Women with higher education were less likely to be assisted by UTBA during 
home delivery. Educated women may understand the need for skilled care attendance during delivery. Besides these, educated women may also have a greater decision making power on seeking health services and have the ability to travel outside home [18, 19]. Women whose husbands have higher education were less likely to be assisted by UTBA during home delivery. Educated husband may be more aware of the benefits of skilled delivery care and more able to reach the appropriate care. They may also be more flexible towards wives mobility and decision making, thus facilitating delivery care seeking.

Wealth quintile has been found as a significant predictor of UTBA assisted home delivery. The finding that women in highest wealth quintile were less likely to deliver at home by UTBA, is similar to other studies $[7,20]$. UTBA assisted home deliveries are deemed affordable for low income families since their payment is negotiable in terms of time and amount [21]. Since households with higher income and living standard are more receptive towards modern health care facilities [22], women belonging to higher wealth quintile may be less likely to have delivered at home by UTBA.

About two in five women (40\%) did not receive any antenatal care services during their last pregnancy. Illiteracy, religious prohibition and lack of knowledge about pregnancy may deter the rural women from receiving ANC services from professional providers. ANC visit has been found as an important predictor in this study. Women who received less antenatal care visit during pregnancy were more likely to choose home delivery. Women, who receive ANC visits, can interact with the health facilities, know the current pregnancy status including possible risks and feel the importance of skilled care attendance during delivery, which in turns discourage them to deliver at home without skilled birth attendant [11]. The association between number of ANC visits and increased uptake of facility delivery has also been reported by other studies in Bangladesh [23,24].

More than two fifth of the women have no access to media as they are residing in rural areas. Absence of electricity in some rural areas and low level of literacy among the rural women may be the major reasons for not having access to media. Mothers who are exposed to media are less likely to deliver at home and assisted by untrained traditional birth attendant. Women exposed to media may be more aware of facility services, know the possible risk of delivering at home and thus feel the importance of skilled care attendance during delivery.

It is recommended that to improve the use of delivery care utilization, the provisions of maternal health care services especially delivery care services may be provided free of cost to the women within the poor wealth quintiles. Women should also be encouraged to continue education to at least secondary level, since this improves the use of delivery care services. Pregnant women should be brought under ANC coverage, since ANC visit is associated with the reduction of home delivery. Finally awareness program through media regarding maternal health can be launched in the rural area. It is also recommended to scale up facility based maternal health care services and increase skilled care attendance of home delivery.

Though the study identified some important factors responsible for UTBA assisted home delivery, the study has some limitations. Findings obtained from this study may not generally applicable to urban settings in Bangladesh as the study was conducted in rural areas. Furthermore, accessibility and availability of health facility were not included in the study. Future study in urban areas including accessibility and availability of health centers will be helpful for finding better outcomes.

\section{Conclusions}

This study indicates that U TBA assistance during home delivery is associated with low education, being poor, low ANC visits and absence of exposure to mass media. To reduce maternal mortality, program managers and policy makers should consider these factors in designing programs and implementing interventions. It is recommended to scale up facility based maternal health care services and increase skilled care attendance of home delivery.

\section{List of Abbreviations}

\author{
ANC - Antenatal Care \\ BDHS - Bangladesh Demographic and Health Survey \\ CI - Confidence Interval \\ DHS - Demographic and Health Survey \\ OR - Odds Ratio \\ UTBA - Untrained Traditional Birth Attendant
}

\section{Competing interests}

The authors declare that they have no competing interests

\section{Acknowledgements}

We thank the Measure DHS for making BDHS 2011 data available to us for analysis. We also like to thank all the mothers who participated in 2011 Bangladesh Demographic and Health Survey.

\section{References}

[1] WHO, UNICEF, UNFPA and The World Bank, Trends in Maternal Mortality: 1990 to 2010, Geneva: WHO, 2010.

[2] Ronsmans, C. and Graham, W.J., "Maternal mortality: who, when, where, and why", Lancet, 368(9542), p:1189-1200, 2006.

[3] Harvey, S.A., Ayabaca, P., Bucagu, M., Djibrina, S., Edson, W.N., Gbangbade, S., McCaw-Binns, A. and Burkhalter, B.R., "Skilled birth attendant competence: an initial assessment in four countries, and implications for the Safe Motherhood movement", International journal of gynaecology and obstetrics, 87(2), p:203210, 2004.

[4] Koblinsky, M.A., Campbell, O. and Heichelheim, J., "Organizing delivery care: what works for safe motherhood?" Bulletin of the World Health Organization, 77(5), p:399-406, 1999.

[5] Kwast, B.E., "Reduction of maternal and perinatal mortality in rural and peri-urban settings: what works?” European journal of obstetrics, gynecology, and reproductive biology, 69(1), p:47-53, 1996.

[6] De Brouwere, V., Tonglet, R. and Van Lerberghe, W., "Strategies for reducing maternal mortality in developing countries: what can we learn from the history of the industrialized West?”, Tropical medicine \& international health, 3(10), p:771-782, 1998. 
[7] Koblinsky, M., Matthews, Z., Hussein, J., Mavalankar, D., Mridha, M.K., Anwar, I., Achadi, E., Adjei, S., Padmanabhan, P. and Marchal, B., "Going to scale with professional skilled care", Lancet, 368(9544), p:1377-1386, 2006.

[8] Montagu, D., Yamey, G., Visconti, A., Harding, A., and Yoong, J., "Where do poor women in developing countries give birth? A multi-country analysis of demographic and health survey data", PloS one, 6(2), 2011.

[9] Campbell, O.M. and Graham, W.J., "Strategies for reducing maternal mortality: getting on with what works". Lancet, 368(9543), p:1284-1299, 2006.

[10] Stephenson, R., Baschieri, A., Clements, S., Hennink, M. and Madise, N., "Contextual influences on the use of health facilities for childbirth in Africa”, American journal of public health, 96(1), $\mathrm{p}: 84-93,2006$.

[11] Gabrysch, S. and Campbell, O.M., "Still too far to walk: literature review of the determinants of delivery service use", BMC pregnancy and childbirth, 9(34), 2009.

[12] Faye, A., Niane, M. and Ba, I., "Home birth in women who have given birth at least once in a health facility: contributory factors in a developing country", Acta obstetricia et gynecologica Scandinavica, 90(11), p:1239-1243, 2011.

[13] Paul, B.K. and Rumsey, D.J., "Utilization of health facilities and trained birth attendants for childbirth in rural Bangladesh: an empirical study”, Soc Sci Med, 54(12), p:1755-1765, 2002.

[14] National Institute of Population Research and Training (NIPORT), Mitra and Associates and Macro International, Bangladesh Demographic and Health Survey 2011, Dhaka, Bangladesh and Calverton, Maryland, USA, 2013.

[15] Titaley, C.R., Dibley, M.J. and Roberts, C.L., "Factors associated with underutilization of antenatal care services in Indonesia: results of Indonesia Demographic and Health Survey 2002/2003 and 2007”, BMC public health, 10(485), 2010.
[16] Shea, O.R. and Kiersten, J., DHS Comparative Report No. 6, The DHS Wealth Index, Calverton, Maryland, USA: Measure DHSORC Macro, 2004.

[17] Bell, J., Curtis, S.L. and Alayón, S., Trends in delivery care in six countries. DHS Analytical Studies No 7, Calverton, Maryland, USA: DHSORC Macro, 2003.

[18] Mekonnen, Y. and Mekonnen, A., "Factors influencing the use of maternal healthcare services in Ethiopia",Journal of health, population and nutrition, 21(4), p:374-382, 2003.

[19] Tann, C.J., Kizza, M., Morison, L., Mabey, D., Muwanga, M., Grosskurth, H. and Elliott, A.M., "Use of antenatal services and delivery care in Entebbe, Uganda: a community survey", BMC pregnancy and childbirth, 7(23), 2007.

[20] Kruk, M.E., Prescott, M.R. and Galea, S., "Equity of skilled birth attendant utilization in developing countries: financing and policy determinants”. American journal of public health, 98(1), p:142147, 2008.

[21] Amooti-Kaguna, B. and Nuwaha, F., "Factors influencing choice of delivery sites in Rakai district of Uganda”, Soc Sci Med, 50(2), $\mathrm{p}: 203-213,2000$.

[22] Navaneetham, K. and Dharmalingam, A., "Utilization of maternal health care services in Southern India”, Soc Sci Med, 55(10), p:1849-1869, 2002.

[23] Vanneste, A.M., Ronsmans, C., Chakraborty, J. and De Francisco, A., "Prenatal screening in rural Bangladesh: from prediction to care”, Health policy and planning, 15(1), p:1-10, 2000.

[24] Pervin, J., Moran, A., Rahman, M., Razzaque, A., Sibley, L., Streatfield, P.K., Reichenbach, L.J., Koblinsky, M., Hruschka, D. and Rahman, A., "Association of antenatal care with facility delivery and perinatal survival - a population-based study in Bangladesh”, BMC pregnancy and childbirth, 12(111), 2012. 